\title{
Assessing Uncertainties in Boundary Layer Transition Predictions for HIFiRE-1 at Non-zero Angles of Attack
}

\author{
Lindsay C. Marek* \\ NASA Johnson Space Center, Houston, Texas, 77058
}

\begin{abstract}
Boundary layer stability was analyzed for the HIFiRE-1 flight vehicle geometry for ground tests conducted at the CUBRC LENS I hypersonic shock test facility and the Langley Research Center (LaRC) 20inch Mach 6 Tunnel. Boundary layer stability results were compared to transition onset location obtained from discrete heat transfer measurements from thin film gauges during the CUBRC test and spatially continuous heat transfer measurements from thermal phosphor paint data during the LaRC test. The focus of this analysis was on conditions at non-zero angles of attack as stability analysis has already been performed at zero degrees angle of attack. Also, the transition onset data obtained during flight testing was at nonzero angles of attack, so this analysis could be expanded in the future to include the results of the flight test data. Stability analysis was performed using the 2D parabolized stability software suite STABL (Stability and Transition Analysis for Hypersonic Boundary Layers) developed at the University of Minnesota and the mean flow solutions were computed using the DPLR finite volume Navier-Stokes computational fluid dynamics (CFD) solver. A center line slice of the 3D mean flow solution was used for the stability analysis to incorporate the angle of attack effects while still taking advantage of the 2D STABL software suite. The $\mathbf{N}$-factors at transition onset and the value of $R e_{\theta} / M_{e}$, commonly used to predict boundary layer transition onset, were compared for all conditions analyzed.

Ground test data was analyzed at Mach 7.2 and Mach 6.0 and angles of attack of $1^{\circ}, 3^{\circ}$ and $5^{\circ}$. At these conditions, the flow was found to be second mode dominant for the HIFiRE-1 slender cone geometry. On the leeward side of the vehicle, a strong trend of transition onset location with angle of attack was observed as the boundary layer on the leeward side of the vehicle developed inflection points at streamwise positions on the vehicle that correlated to angle of attack. Inflection points are a strong instability mechanism that lead to rapid breakdown and transition to turbulence. The transition onset location on the windward side of the vehicle displayed no trend with angle of attack or freestream Reynolds number and transition was observed farther down the vehicle than observed on the leeward side of the vehicle. In analysis of both windward and leeward sides of the vehicle, use of the $\mathrm{N}$ factor methodology to develop trends to predict boundary layer transition onset showed improvements over the $R e_{\theta} / M_{e}$ empirical correlation methodology. Stronger correlations and less scatter in the data were observed when using the $\mathbf{N}$ factor method for these cases.
\end{abstract}

\section{Introduction}

The HIFiRE-1 test program was developed to advance research in weapons systems by demonstrating instrumentation capabilities on a flight vehicle ${ }^{1}$. Laminar to turbulent transition and turbulent separated shock-boundary layer interaction were the flight objectives chosen to demonstrate those capabilities and the HIFiRE-1 flight geometry was designed with these test objectives in mind. First, a $7^{\circ}$ half-angle cone with a $2.5 \mathrm{~mm}$ nose radius was selected for the fore body and instrumented to observe natural and tripped transition to turbulence. At the aft end of the cone was a straight, cylindrical section to allow for the turbulent flow to separate, and then a $33^{\circ}$ flare was attached at the end of the cylinder and was densely instrumented to obtain data on the resulting turbulent separated shock-boundary layer interaction region. The flight geometry can be seen in Figure 1.

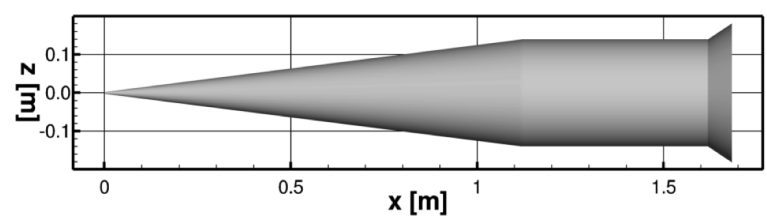

Figure 1. HIFiRE-1 Flight Geometry.

${ }^{*}$ Aerospace Engineer, Applied Aerosciences and Computational Fluid Dynamics Branch, AIAA Member. 


\section{A. Review of Ground Testing}

The HIFiRE-1 geometry was tested in two ground test facilities. The CUBRC LENS I hypersonic shock test facility in Buffalo, New York tested the full scale geometry at flight Reynolds numbers to duplicate the reentry trajectory. ${ }^{2}$ The Langley Research Center tested a scaled flight geometry in the 20-inch Mach 6 Tunnel. ${ }^{3}$ Information on both of these tests can be found in other publications in more detail, so only a brief overview will be covered here.

During the test at the CUBRC LENS I facility, two entries were completed with varying nose radii and flare angles to optimize the geometry to meet the flight test objectives. Angles of attack of $0^{\circ}, 1^{\circ}$, and $5^{\circ}$ were also tested. Discrete thin film sensors were placed at four azimuthal locations to capture the transition behavior at angles of attack. Runs selected from the CUBRC test series for this analysis consisted only of runs where the geometry configuration matched the configuration flown during the flight test.

The LaRC test series in the 20-inch Mach 6 Tunnel tested two models each with two different nose radii (four models in total). The first was a $20 \%$ scale model of the entire flight vehicle and the second was a $35 \%$ scale $7^{\circ}$ half-angle cone without the cylinder and flare. Thermal phosphor paint was used to capture spatially continuous heat transfer data to gain global transition behavior at $0^{\circ}, \pm 3^{\circ}$, and $\pm 5^{\circ}$ angles of attack. The runs at positive and negative non-zero angles of attack at each run condition were designed to capture transition data on both windward and leeward sides of the vehicle.

While many runs were completed for the tests conducted at CUBRC and LaRC to meet different test objectives and to gather transition data for a variety of conditions, Table 1 lists the runs and conditions chosen for the analysis in this publication. These conditions were chosen because data was gathered at non-zero angles of attack where transition was observed. Runs were chosen to get transition information on both the windward and leeward sides of the vehicle, however, not all cases transitioned on both sides of the vehicle.

Table 1. Ground Test Data Analyzed

\begin{tabular}{ccccc} 
Facility & Run & Mach & $\mathrm{Re} / \mathrm{m}\left[\times 10^{-6}\right]$ & $\alpha\left[^{\circ}\right]$ \\
\hline CUBRC & 10 & 7.152 & 10.23 & 1 \\
CUBRC & 17 & 7.140 & 10.43 & 1 \\
LaRC & $10 / 22$ & 5.977 & 8.11 & 3 \\
LaRC & $12 / 24$ & 6.007 & 13.38 & 3 \\
LaRC & $63 / 65$ & 6.028 & 18.68 & 3 \\
LaRC & $16 / 28$ & 5.977 & 8.02 & 5 \\
LaRC & $19 / 29$ & 6.006 & 13.03 & 5 \\
LaRC & $64 / 30$ & 6.028 & 18.63 & 5 \\
CUBRC & 11 & 7.176 & 10.65 & 5 \\
CUBRC & 13 & 7.155 & 10.37 & 5
\end{tabular}

Also of importance is the nose radius of each of the models because the nose radius plays a significant role in the stability of the boundary layer. The model used for the CUBRC test was at the flight scale, so the nose radius of $2.5 \mathrm{~mm}$ was the same for the test article in the tunnel as well as the flight vehicle. While the LaRC test series used a scaled model, the nose radius was not directly scaled the same amount. The LaRC tests used models with a $1.19 \mathrm{~mm}$ nose radius and models with a $2.1 \mathrm{~mm}$ nose radius for their testing. When scaled up to the flight geometry, this would result in a blunter nose tip than the CUBRC test article and the flight geometry. A blunt nose tip serves to stabilize the boundary layer and delay transition in most cases and must be taken into account in the analysis presented in this paper.

\section{B. Empirical Transition Prediction Methods: Correlation and Physics-Based Analyses}

Predicting boundary layer transition is a difficult problem that many researchers have spent careers trying to solve. Boundary layer transition is a complex process that can be governed by fluctuations in freestream properties, surface roughness, surface curvature, boundary layer properties and many other geometric and flow phenomena. While many advances have been made in determining the characteristics of a boundary layer and its stability, none have the capability to predict transition as a stand-alone method. ${ }^{4}$ Even more advanced physics-based methods still require 
transition onset information from testing. Depending on the application, a strong case can be made for empirical correlations based on boundary layer properties and equally strong cases can be made for physics-based methods of analyzing boundary layer stability to predict transition. Empirical correlations based on boundary layer properties are very useful in engineering level vehicle design or performance evaluations when simple and effective tools are needed to assess a design or situation quickly ${ }^{5}$, while physics-based methods are useful for understanding the mechanisms that cause boundary layer transition as well as how the different modes interact with each other or the amplitude of disturbances grows or decays.

One popular method for predicting the onset of boundary layer transition empirically correlates transition onset location observed in test data to boundary layer properties at that location from a computational simulation. However, empirical correlations based on boundary layer properties are only valid for similar geometries at similar conditions and usually cannot be generalized to multiple different geometries or test conditions. ${ }^{6}$ Two popular boundary layer properties used to predict the onset of boundary layer transition on smooth bodies are $R e_{\theta}$, the Reynolds number based on the momentum thickness of the boundary layer, and $R e_{\theta} / M_{e}$, the momentum thickness Reynolds number divided by the Mach number at the edge of the boundary layer. Transition onset location can also be correlated to many other boundary layer properties, some of which are addressed by Campbell, et. al. ${ }^{5}$, but only $R e_{\theta} / M_{e}$ will be addressed in the work presented here.

While correlations based on boundary layer properties can be useful for engineering problems, they do not capture much of the physics of the boundary layer and they do not take into consideration many of the variables affecting the growth of disturbances in the boundary layer which can lead to transition onset. Physics-based methods such as the $e^{N}$ method and the parabolized stability equations (PSE) have the ability to incorporate the physics governing the mean flow to track the growth of disturbances within the boundary layer that may lead to transition. A higherfidelity mechanism based approach to boundary layer stability could provide more accurate methods for predicting boundary layer transition onset. A better understanding of the transition mechanisms, however, is required to validate these methods. Many conventional wind tunnels produce transition data that is highly influenced by the noise propagated from the turbulent boundary layers that develop on the tunnel walls. ${ }^{7}$ As a result, transition data obtained in quiet tunnels where the boundary layer on the nozzle walls remains laminar and radiated noise and freestream fluctuations are greatly reduced provides validation data for transition from other instability mechanisms. Measuring those instability mechanisms presents its own set of challenges, though. Reshotko ${ }^{7}$ outlines a set of guidelines than can be used for setting up experiments to gather transition data. More and better data on the mechanisms that cause transition from a wide variety of sources would be very beneficial to advancing boundary layer stability predictions and could be used to validate and inform the physics-based models currently used.

It should be emphasized at this point that it is not the purpose of this publication to say which method is better for all applications. This paper addresses two methods on a single geometry and it is not assumed that the results can accurately be extrapolated to another geometry or other conditions.

\section{Methodology}

All ground test conditions selected for this analysis were at a non-zero angles of attack to examine the effects of angle of attack on the boundary layer stability and transition onset location. The boundary layer transition data obtained during the HIFiRE-1 flight test was also at non-zero angle of attack for both ascent and descent measurements, so future analysis of the flight data could include this methodology.

The stability analysis was completed using the stability modeling software, STABL (Stability and Transition Analysis for Hypersonic Boundary Layers), developed at the University of Minnesota, which uses the linear PSE (parabolized stability equation) method in PSE-Chem. ${ }^{8}$ This method uses a laminar basic state solution and assumes the small perturbations in flow properties of the form of Equation 1 for pressure, density, temperature, and three components of velocity. 


$$
\begin{aligned}
p & =P+p^{\prime} \\
\rho & =\tilde{\rho}+\rho^{\prime} \\
T & =\tilde{T}+T^{\prime} \\
\mathbf{u} & =\mathbf{U}+\mathbf{u}^{\prime}
\end{aligned}
$$

The perturbed variables are then substituted into the boundary layer equations and the system of equations is parabolized to obtain the Parabolized Stability Equations (PSE), which reduce to Equation 2 for the two-dimensional case of importance here.

$$
\left(L+\epsilon L^{\prime}\right) \mathbf{q}+\epsilon M_{1} \frac{\partial \mathbf{q}}{\partial \xi}=\mathbf{r}
$$

In the above equation, $\mathbf{q}$ is the vector of flow variables, $L, L^{\prime}$, and $M_{1}$ are operators in the off-body direction, $\xi$ allows for slow variations in the streamwise direction, and $\mathbf{r}$ on the right hand side includes the non-linear terms. The eigenvalues of the system are found to determine the real and complex disturbance frequencies that are used to calculate the amplification of each disturbance. A detailed derivation of the PSE methodology can be found in Parabolized Stability Equations by Thorwald Herbert ${ }^{9}$.

The growth and decay of those imposed perturbations are then tracked down the length of the body as a function of disturbance frequency. The amplitude of each disturbance is integrated using Equation 3 to determine the $\mathrm{N}$ factor at each point along the body and a maximum $\mathrm{N}$ factor curve is created. Figure 2 shows a sample maximum $\mathrm{N}$ factor curve.

$$
N=\int_{x_{0}}^{x} \frac{1}{A} \frac{d A}{d x} d x=\ln \frac{A}{A_{0}}
$$

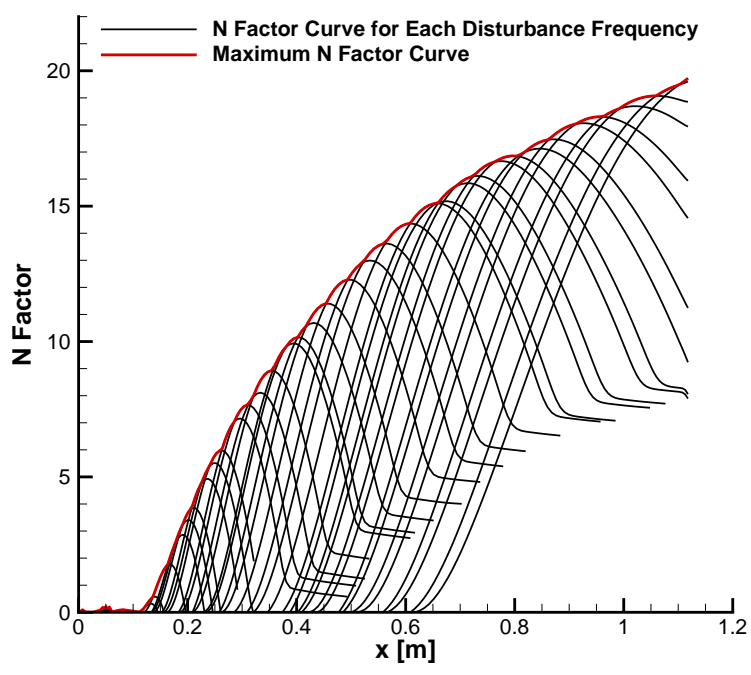

Figure 2. Maximum N Factor Curve

Mean flow solutions were computed using the DPLR finite volume Navier-Stokes flow solver ${ }^{10}$ on a grid with $\approx 8.5$ million grid cells. Viscosity was modeled using Sutherland's Law and thermal conductivity was computed assuming a constant Prandlt Number. For simplicity, the aft end of the geometry was excluded from the analysis and only a cone was simulated. The three-dimensional nature of the flow field for a cone at angle of attack can be seen in the surface heat transfer and surface shear lines in Figure 3. On the leeward side of the vehicle, two vortices develop as the flow diverges from the windward side. The vortices pull high momentum fluid from high in the boundary layer down towards the surface of the vehicle which modifies the mean flow solution and introduces instabilities into the boundary layer. This must be noted as it will be important in later analysis. 


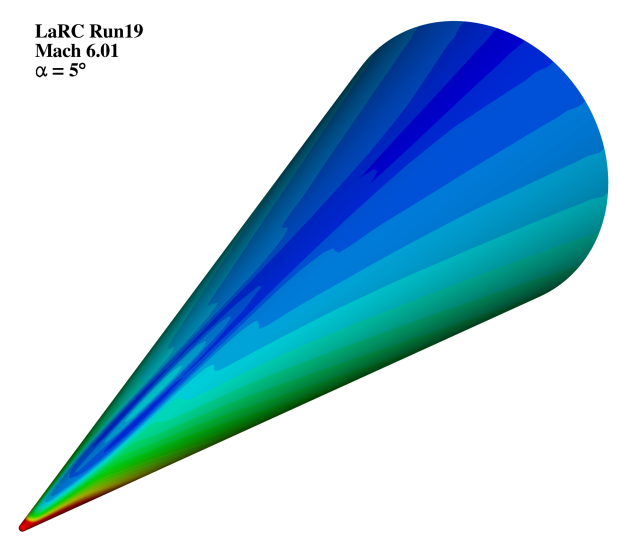

(a) Surface heat transfer

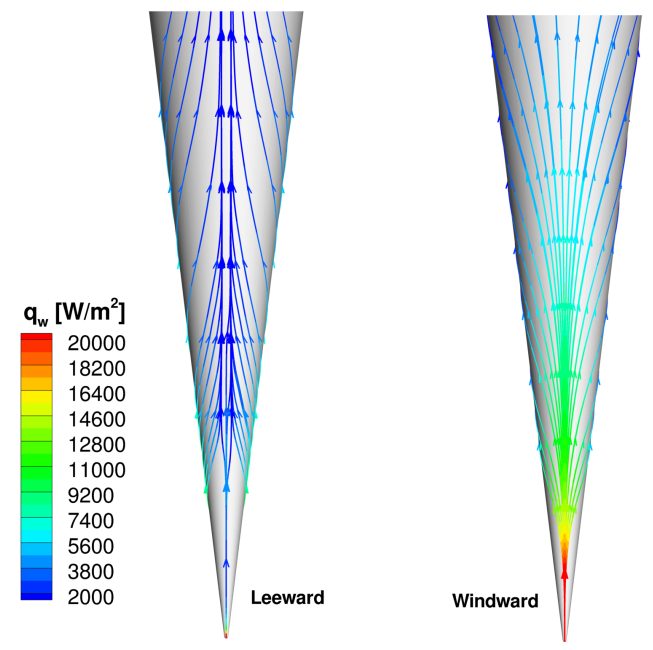

(b) Surface shear lines

Figure 3. Solution at $5^{\circ}$ angle of attack.

The STABL software used for analyzing the boundary layer stability is a 2D code, but a cone at a non-zero angle of attack produces a 3D flow field. For this reason, a centerline slice was extracted from a 3D mean flow solution for the stability analysis of each condition. The centerline slice contained 157 cells in the off-body direction and approximately 75-100 of those cells resolved the boundary layer. The grid was split at the stagnation point to allow for analysis of the windward and leeward stability separately. The flow on the centerline pitch plane used for these analyses can be seen in Figure 4. The frequencies of the disturbances in the boundary layer are largely affected by the thickness of the boundary layer, so the difference in the thickness of the boundary layer from the leeward side to the windward side should be noted. Since the disturbance frequency is inversely proportional to the boundary layer thickness, ${ }^{11}$ larger disturbance frequencies would be expected on the windward side of the vehicle.

$$
\omega \propto \frac{1}{\delta}
$$

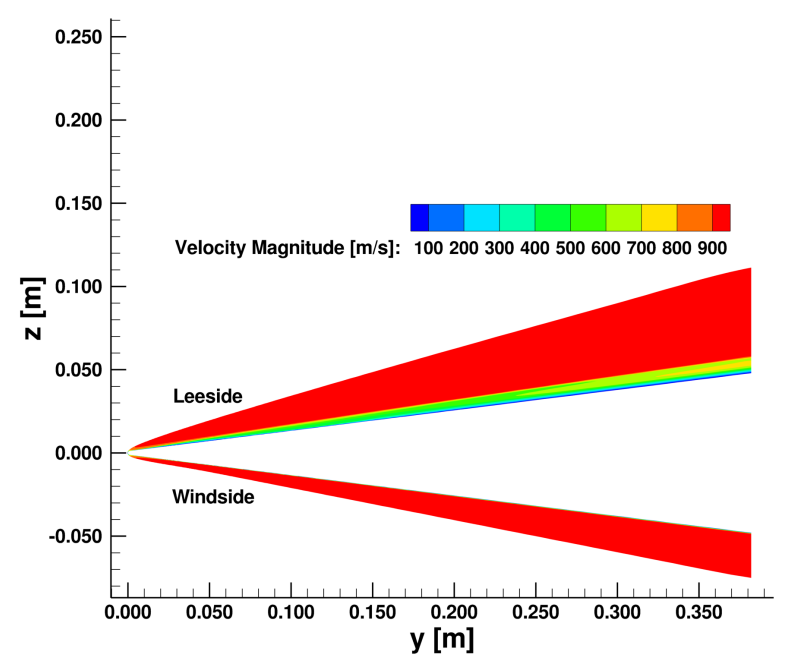

Figure 4. Centerline mean flow solution used for stability analysis. 
This methodology, however, has limitations. As previously mentioned, the flow field around a cone at angle of attack is very three-dimensional. Approximating the three-dimensional flow field with a two-dimensional centerline slice ignores many effects including curvature, crossflow, and other three-dimensional effects that have significant effects on the transition behavior of a boundary layer. As a result, the analysis presented in this paper assumes that a two-dimensional slice along the plane of symmetry of a three-dimensional solution provides a representative flow field for stability analysis by minimizing the three-dimensional effects. This assumption will introduce errors into the analysis; the quantification of which may be addressed in future work.

Transition onset prediction using the $\mathrm{N}$ factor method described is semi-empirical. The maximum $\mathrm{N}$ factor curve is computed for each condition and the $\mathrm{N}$ factor at transition must be determined by comparison to the measured transition onset location from experiment. The transition onset location for each of the ground test conditions from the CUBRC test series was computed using the method of zero intermittency. ${ }^{12}$ This method uses the time averaged heat transfer data from the discrete thin film gauges located on the test article to extrapolate a trend line through the transitional data to the point of zero intermittency, or the point where this line intersects with the laminar CFD prediction. This method was chosen because time traces of the thin film gauges for the CUBRC ground tests, which can provide more isight into the onset of transition, were not available. The uncertainty associated with the streamwise location of transition onset was the distance between the last gauge showing laminar behavior and the first gauge to deviate from the laminar prediction and exhibit transitional behavior.

Analyzing the transition onset location from the LaRC relied more on engineering judgement because the heat transfer data obtained from this test was spatially continuous. The point of transition onset was chosen where the heat transfer data began to change slope and increase above the laminar prediction.

Figure 5 shows how each of these methods was used to select the best estimate transition onset location and apply a range of transition onset uncertainty. The transition onset range shown in these figures gives the uncertainty in the streamwise direction along the body associated with the transition onset location described previously. It should be noted that the determination of the transition onset location and the associated uncertainties is very subjective. This publication presents only the author's analysis. An independent analysis would likely produce different $\mathrm{N}$ factors based on transition location selection, which may or may not have an impact on the transition onset location predicted by the method.

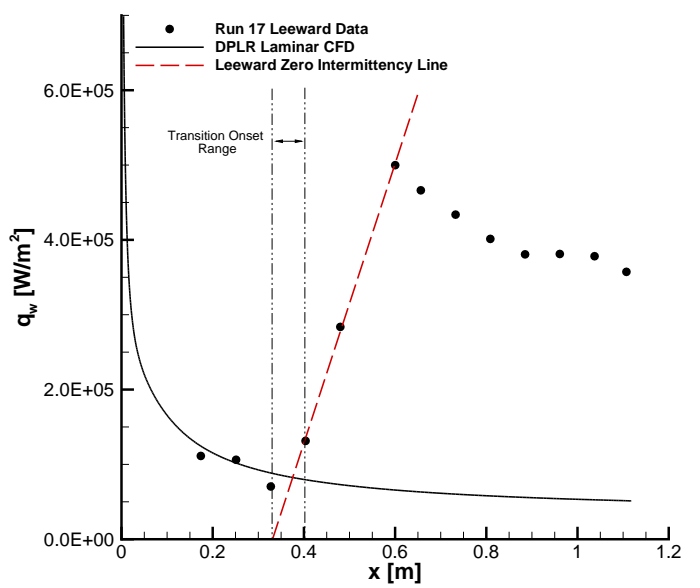

(a) CUBRC Discrete Measurements with Zero Intermittency Line.

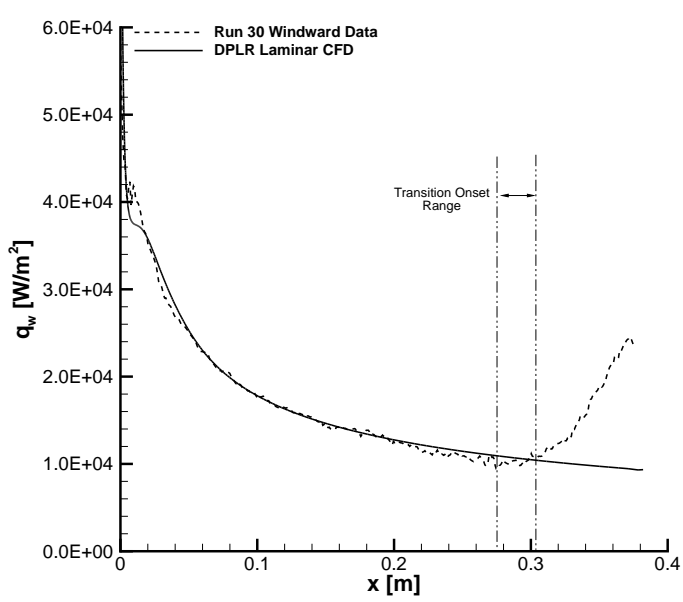

(b) LaRC Continuous Measurements with Observed Transition Onset.

Figure 5. Transition onset determination methods. 


\section{Results}

Conditions were analyzed for the HIFiRE-1 geometry at ground test conditions from the CUBRC LENS I test facility as well as the Langley Research Center (LaRC) 20-inch Mach 6 Tunnel. The conditions analyzed can be found in Table 1, where the two run numbers from the LaRC tests denote the leeward and windward runs, respectively, since the temperature sensitive paint data at that facility was gathered only on one side of the vehicle for each run of this test. For all LaRC test data analyzed in this publication, the $1.19 \mathrm{~mm}$ nose radius was used.

For each run, the transition onset location obtained from the experimental data was compared to the maximum $\mathrm{N}$ factor curve computed by the boundary layer stability analysis on the centerline slice of the solution. The $\mathrm{N}$ factor was extracted at three streamwise locations for each run on the windward and leeward sides: the best estimated transition onset location and the associated positive and negative uncertainties. $R e_{\theta} / M_{e}$ was also extracted at the same locations for comparison of the empirical transition prediction methodology to the physics-based $\mathrm{N}$ factor method. Trends of the transition onset location values of $\mathrm{N}$ factor and $R e_{\theta} / M_{e}$ with freestream Reynolds number and angle of attack were examined.

\section{A. Leeward Analysis}

Analysis of the boundary layer on the leeward side of the vehicle showed that it was inherently less stable than the windside boundary layer. Flow structures developed in the mean flow solutions that greatly affected the boundary layer stability. The flow did not separate, but the angle of attack effects included a 'mushroom' of fluid build up along the centerline in the spanwise direction that caused inflection points within the velocity profile in the boundary layer. Low momentum fluid from low within the boundary layer was brought up into the higher speed flow outside the boundary layer and higher momentum fluid was pushed down towards the surface. The 'mushroom' and the resulting inflection points in the boundary layer can be seen in the 3D mean flow solution in Figure 6. Inflection points in the boundary layer are a strong instability mechanism that cause rapid transition to turbulence. Data showed that transition occurred shortly downstream of the location where inflection points in the boundary layer were first observed.

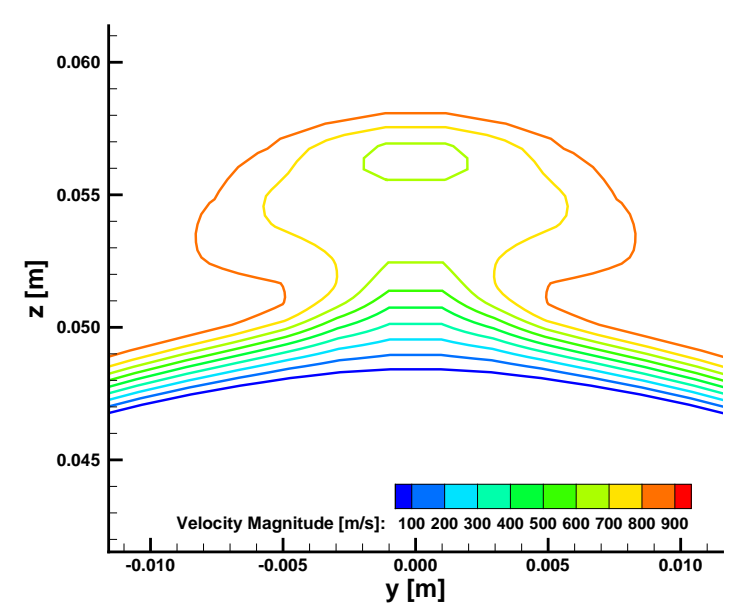

(a) Low momentum 'mushroom' developing in the spanwise direction on the lee side of the vehicle.

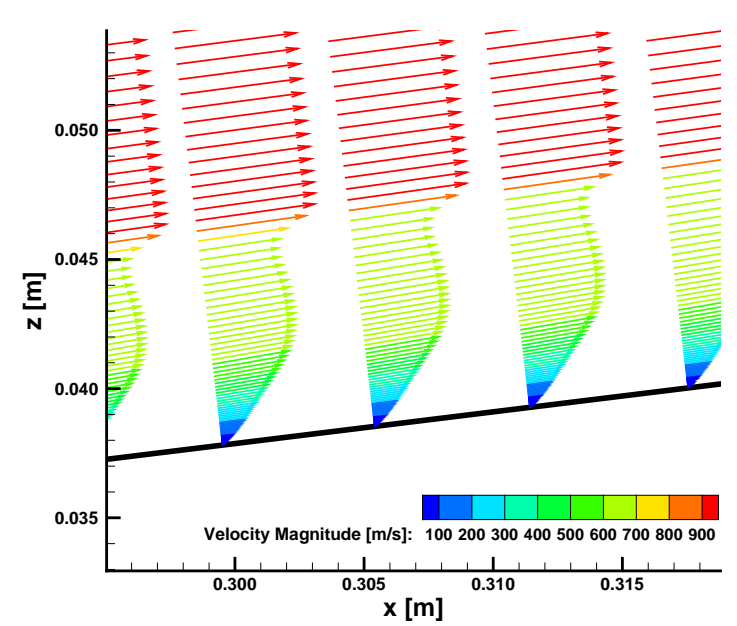

(b) Inflection points in the boundary layer profile along the centerline in the streamwise direction.

Figure 6. Leeward Instability Mechanisms

The location of transition onset observed during ground testing was compared to the $\operatorname{Re}_{\theta} / M_{e}$ computed in the mean flow simulation as well as the maximum $\mathrm{N}$ factor curve generated by STABL for each condition. The values of $R_{\theta} / M_{e}$ compared with the test data for CUBRC Run 17 and LaRC Run 19 on the leeward side of the vehicle can be seen in Figure 7. Similarly, the $\mathrm{N}$ factor comparison for the same runs can be seen in Figure 8. In both of the comparisons for the LaRC test data, there is a discrepancy between the heat transfer data obtained using the thermal 
phosphor paint and the laminar heat transfer computed in the mean flow solution. This is due to the data resolution of the thermal phosphor paint which was not able to capture the increased heating on the centerline due to the vortices rolling up from the windward side of the vehicle. In these cases, the transition onset location was selected where the heat transfer data began to deviate from the expected heat transfer behavior without regard to the vortices, similar to the laminar heat transfer profile computed by DPLR shown in Figure 7a.

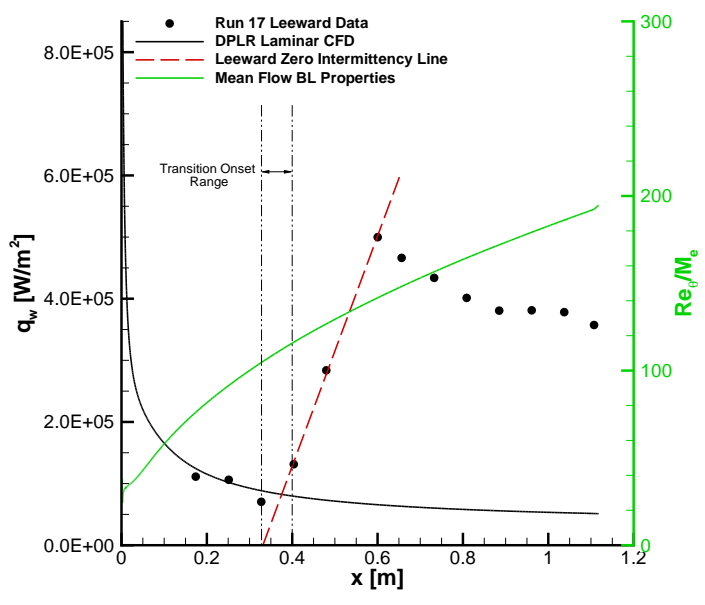

(a) CUBRC Run 17 Leeward Data

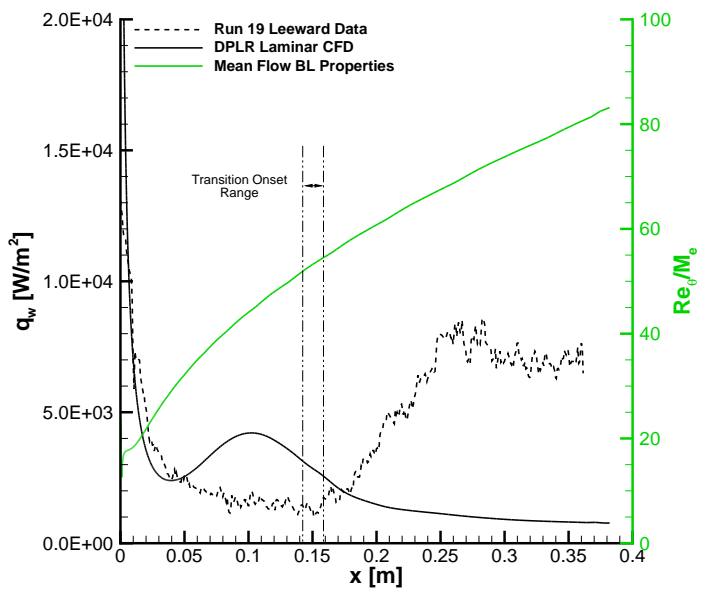

(b) LaRC Run 19 Leeward Data

Figure 7. $R e_{\theta} / M_{e}$ at Transition Onset

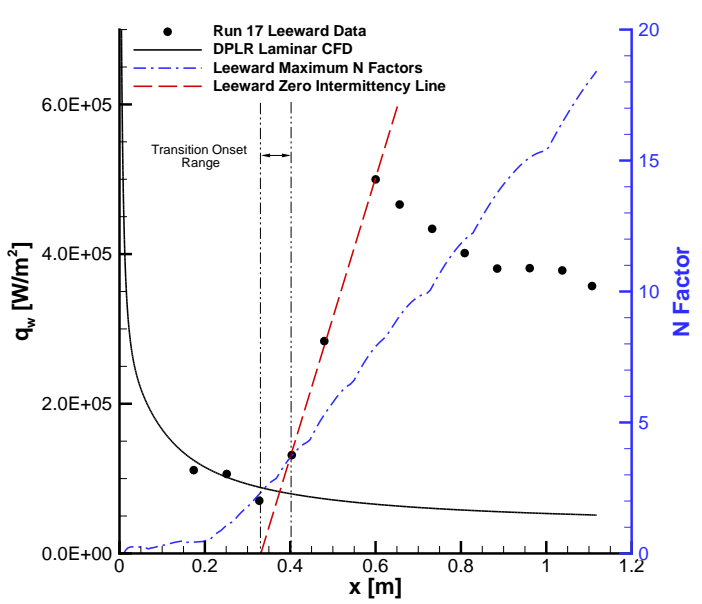

(a) CUBRC Run 17 Leeward Data

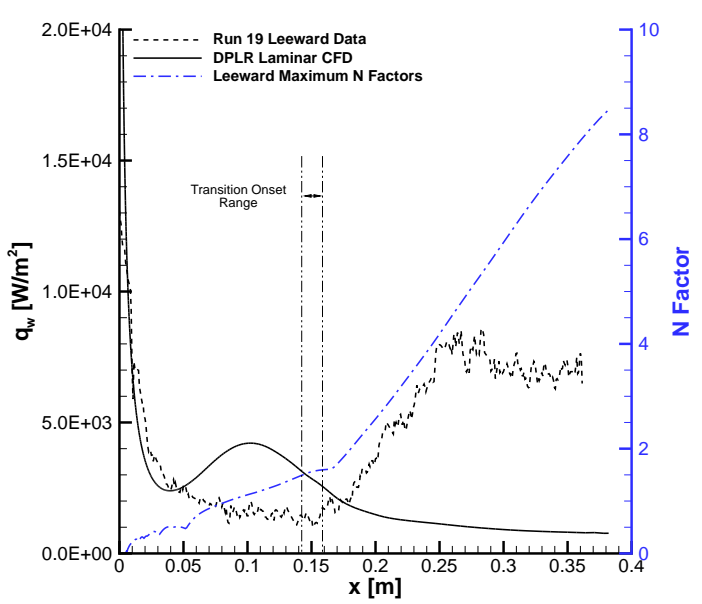

(b) LaRC Run 19 Leeward Data

Figure 8. N Factor at Transition Onset

The value of $\operatorname{Re}_{\theta} / M_{e}$ and $\mathrm{N}$ factor at the transition onset location determined from the data on the leeward side was complied for all ground test runs to examine trends and assess uncertainties associated with each method. Trends with freestream Reynolds number and angle of attack were examined, but results showed only trends with angle of attack on the leeward side. Two runs from the CUBRC data set at $5^{\circ}$ angle of attack were excluded from this analysis because the gauge resolution on the leeward side of the vehicle was not high enough to capture any laminar data before transition onset. This made determining the transition onset location impossible based on the data available using the methods described here. The angle of attack trends can be seen in Figure 9 . 


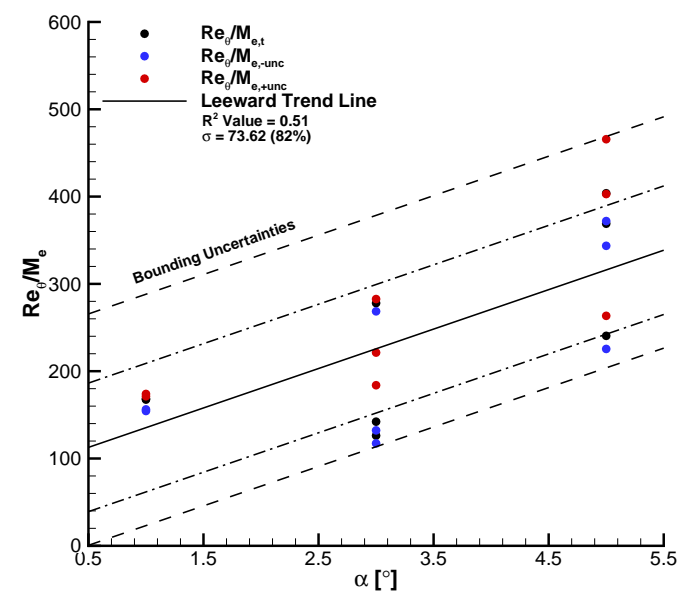

(a) $R e_{\theta} / M_{e}$ Method

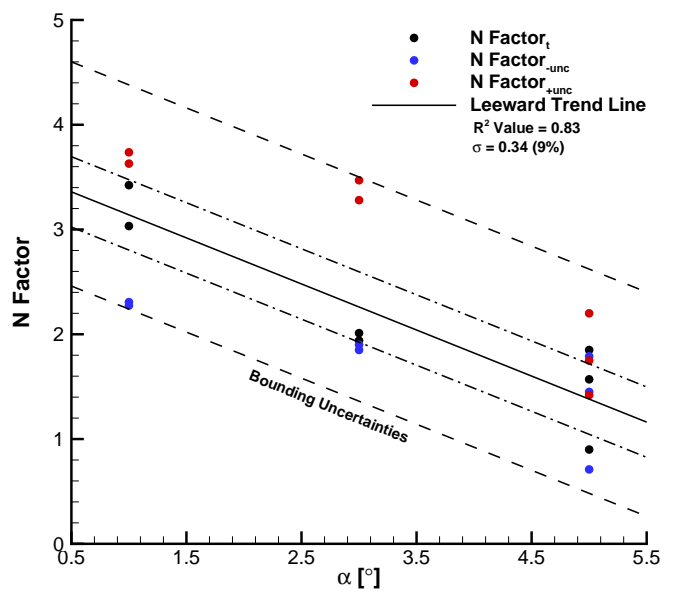

(b) N Factor Method

Figure 9. Correlations of leeward data obtained at transition onset

Figure 9a shows the computed $R e_{\theta} / M_{e}$ at transition onset as a function of angle of attack. This empirical correlation methodology shows a strong trend with angle of attack, but wide scatter in the data. The mean value of the data obtained at transition onset results in an $R^{2}$ value of only 0.51 , which emphasizes this scatter. The scatter in the data also increases with the angle of attack so uncertainty also increases with angle of attack. Ignoring this fact and placing 1- $\sigma$ uncertainties on the data results in a value that is $82 \%$ of the mean. These 1- $\sigma$ uncertainties do not cover the spread of the data and bounding uncertainties result in an asymmetric uncertainty distribution. This may mean that the uncertainty in the data does not follow a Gaussian distribution, but further analysis of the uncertainties is needed.

A similar correlation developed using the $\mathrm{N}$ factor at transition significantly reduces the uncertainties associated with predicting the transition using this method. Figure $9 \mathrm{~b}$ also shows a strong trend of the $\mathrm{N}$ factor at transition onset with angle of attack but the scatter in the data is much less than that seen in the $R_{\theta} / M_{e}$ comparison. This can be seen by a larger $R^{2}$ of 0.83 on the mean of the data and the 1- $\sigma$ uncertainties on the mean span most of the data obtained at transition onset as well as some of the uncertainties associated with determining the transition onset location. The 1- $\sigma$ uncertainty is only $9 \%$ of the mean value for the $\mathrm{N}$ factor method, and a bounding uncertainty placed on all data is more symmetric than the $R e_{\theta} / M_{e}$ correlation which could mean the uncertainties in this case follow a Gaussian distribution more closely and be better predicted.

\section{B. Windward Analysis}

Ground test data on the windward side of the vehicle was compared to the maximum $\mathrm{N}$ factor curve and the value of $R e_{\theta} / M_{e}$ and these values were extracted at the observed transition onset location and at the endpoints of the positive and negative uncertainties as in the leeward data analysis. The windward data showed higher heat transfer rates than the leeward side, but no obvious three-dimensional effects such as the low-momentum 'mushroom' or boundary layer inflection points. The windward ray of gauges in the CURBC test had less gauge resolution than the ray of gauges on the leeward side of the vehicle which causes greater uncertainty in the transition onset location on the windward side. In many cases in the LaRC tests, the flow remained laminar for the entire length of the body. This phenomenon is counterintuitive, but has been seen in many investigations of transition on cones at angle of attack and is attributed to three-dimensional effects in the flow. ${ }^{14}$ Figure 10 shows the windward data for Run 11 from the CUBRC test series and Run 19 from the LaRC test. The maximum $\mathrm{N}$ factor curves for the LaRC data were also much lower in magnitude than the maximum $\mathrm{N}$ factor curves computed for the CUBRC data. 


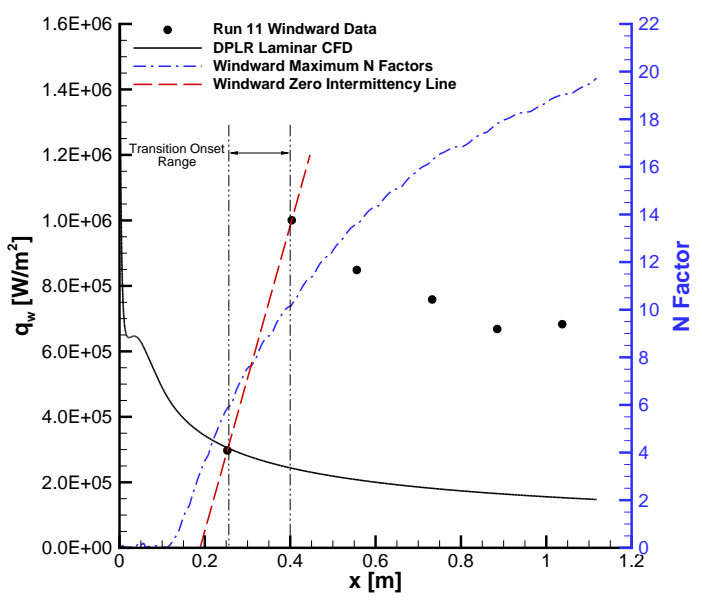

(a) CUBRC Run 11

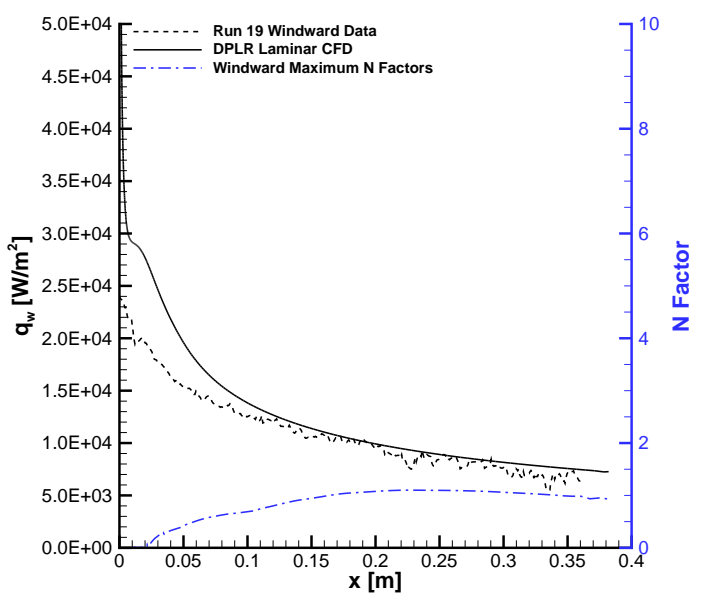

(b) LaRC Run 19

Figure 10. Windward Data Comparisons

No trends of $\mathrm{N}$ factor or $R e_{\theta} / M_{e}$ with freestream Reynolds number or angle of attack were observed, so the uncertainties assessed on the windward side of the vehicle were examined without regard for a physical flow or geometric parameter. The mean value of the data extracted at the transition onset location and 1- $\sigma$ and bounding uncertainties were examined. Comparisons for the $\mathrm{N}$ factor at transition onset for the LaRC data and the CUBRC data differed by a factor of at least four based on the two LaRC runs which exhibited transitional behavior on the windward side. As a result, the LaRC data and CUBRC data were analyzed separately. More than two transitional data points from the LaRC test series should be examined to create any correlations and draw meaningful conclusions. As a result, only data from the CUBRC test is presented here.

The results of the $R_{\theta} / M_{e}$ empirical correlation for the CUBRC data, seen in Figure 11a, shows more scatter in the data and larger uncertainties than those observed in the evaluation of the $\mathrm{N}$ factors computed at transition (Figure 11b). The 1- $\sigma$ uncertainty in the $R e_{\theta} / M_{e}$ methodology is reduced when using the $\mathrm{N}$ factor method. Opposite of the findings on the leeward side of the vehicle, the bounding uncertainties in the $R e_{\theta} / M_{e}$ empirical methodology are more symmetric than those for the $\mathrm{N}$ factor methodology. A more detailed analysis of the distribution of the uncertainties around the mean value will be required to address this finding.

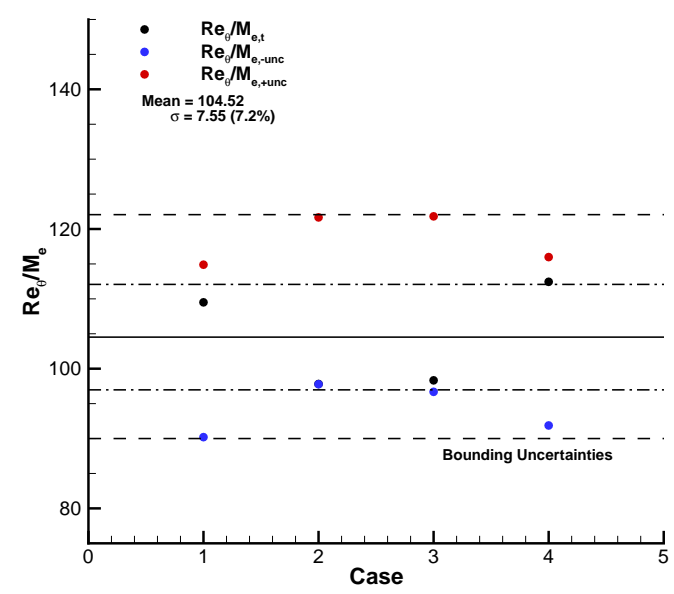

(a) $R e_{\theta} / M_{e}$ Method

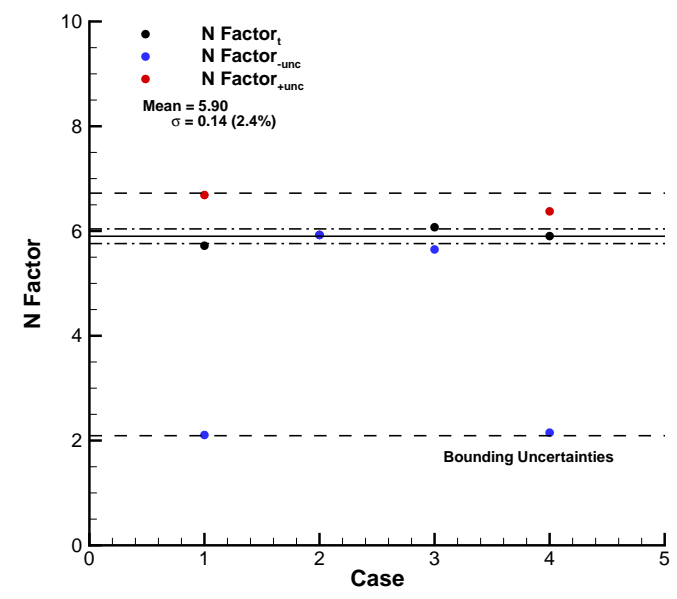

(b) N Factor Method

Figure 11. Correlations of windward data obtained at transition onset from CUBRC data. 
For the LaRC windward data, two runs showed transitional behavior - Run 30 at $5^{\circ}$ angle of attack and Run 65 at $3^{\circ}$ angle of attack. The $\mathrm{N}$ factors at transition were 1.5 and 0.6 , respectively, which were significantly less than the $\mathrm{N}$ factors at transition computed from the CUBRC conditions. Additionally, for all of the other runs in the LaRC data set that did not show transition in the heat transfer data on the windward side, the maximum $\mathrm{N}$ factor curves did not reach above 2. The difference in the relative nose radius between the CUBRC and LaRC test articles previously discussed could explain this difference in part, but many other factors could be contributing to the large difference in $\mathrm{N}$ factors.

\section{Conclusion}

Ground test data for the HIFiRE-1 geometry from the CUBRC LENS I reflected shock tunnel and Langley Research Center 20-inch Mach 6 Tunnel was used to examine the boundary layer stability and transition onset characteristics at non-zero angles of attack. Discrete heat transfer measurements from thin film gauges from the CUBRC data sets and spatially continuous heat transfer measurements from thermal phosphor paint during the LaRC tests were used to determine transition onset location. The transition onset location was used to extract the boundary layer property $R e_{\theta} / M_{e}$, which is commonly used to predict boundary layer transition in empirical correlations, from the laminar mean flow solution and the maximum $\mathrm{N}$ factor computed from the boundary layer stability analysis. Windward and leeward sides of the vehicle were analyzed separately.

On the leeward side of the vehicle, a strong trend of both transition onset indication parameters with angle of attack was observed. This correlates strongly to the development of inflection points in the boundary layer that are a strong instability mechanism and lead to rapid breakdown to turbulence. The windward side of the vehicle transitioned farther down the body than on the leeward side, but no trends were observed with angle of attack or freestream Reynolds number. Using both methods of predicting boundary layer transition onset, uncertainty analysis was performed. The $\mathrm{N}$ factor method in all cases presented here showed a stronger correlation with less scatter in the data and smaller uncertainties than the $R e_{\theta} / M_{e}$ method. However, bounding uncertainties were asymmetric about the mean in some cases, and a more detailed analysis that addresses the distribution of the uncertainty around the data must be performed.

In general, the maximum $\mathrm{N}$ factor curves computed for the LaRC test data had lower values of $\mathrm{N}$ factor than the curves generated for the CUBRC test data. On the windward side of the vehicle, the $\mathrm{N}$ factors between the two facilities differed by at least a factor of four. This could be due to the difference in the relative nose radius of the vehicles. The LaRC test article had a larger nose radius relative to the length of the vehicle than the CUBRC test article and the flight vehicle. A larger nose radius has been shown to stabilize the boundary layer and delay transition, so this could be contributing to the differences in the computed $\mathrm{N}$ factors. Further analysis would be required to determine the cause of the discrepancy with more certainty.

The results of this investigation showed a decrease in uncertainty when using a physics-based transition prediction methodology when compared to an empirical correlation based on $R e_{\theta} / M_{e}$ for the HIFiRE-1 slender cone geometry at ground test conditions. The analysis at non-zero angles of attack presented here could be expanded in the future to incorporate the results of the HIFiRE-1 flight test as most of the transition data obtained during the

flight was at non-zero angles of attack. Including the flight data in future analysis would also begin to address the issue of flight traceability in boundary layer transition onset predictions. 


\section{Acknowledgments}

The author would like to acknowledge the support of the Exploration Technology Development and Demonstration (ETDD) Program, managed at NASA-Glenn Research Center. The work documented herein was performed as part of ETDD's Entry, Descent and Landing (EDL) Technology Development Project, which is managed at NASALangley Research Center and supported by NASA-Ames Research Center, NASA-Johnson Space Center, and the Jet Propulsion Laboratory.

Additionally, the author would like to acknowledge Karen Bibb from Langley Research Center and Tim Wadhams from CUBRC for their assistance in gathering and interpreting the ground test data obtained at their respective facilities.

\section{References}

${ }^{1}$ Kimmel, R., Adamczak, D., Gaitonde, D., Rougeux, A., and Hayes, J., "HIFiRE-1 Boundary Layer Transition Experiment Design,” 45th Aerospace Sciences Meeting \& Exhibit, AIAA Paper 2007-0534, Jan. 2007.

${ }^{2}$ Wadahms, T., MacLean, M., Holden, M., and Mundy, E., "Ground Test Studies of the HIFiRE-1 Transition Experiment Part 1: Experimental Results," Journal of Spacecraft and Rockets, Vol. 45, No. 6, 2008.

${ }^{3}$ Berger, K., Greene, F., Kimmel, R., Alba, C., and Johnson, H., "Aerothermodynamic Testing and Boundary-Layer Trip Sizing of the HIFiRE Flight 1 Vehicle,” Journal of Spacecraft and Rockets, Vol. 45, No. 6, 2008.

${ }^{4}$ Stetson, K., "On Predicting Hypersonic Boundary Layer Transition," Air Force Wright Aeronautical Laboratories Technical Memo, AFWAL-TM-87-160-FIMG, March 1987.

${ }^{5}$ Campbell, C., King, R., Berry, S., Kegerise, M., Horvath, T., "Roles of Engineering Correlations in Hypersonic Entry Boundary Layer Transition Prediction," 48th AIAA Aeroscience Meeting Conference, AIAA Paper 2010-0247, Jan. 2010.

${ }^{6}$ Schneider, S., "Flight Data for Boundary-Layer Transition at Hypersonic and Supersonic Speeds," Journal of Spacecraft and Rockets, Vol. 36, No. 1, 1999.

${ }^{7}$ Reshotko, E., "Transition Issues for Atmospheric Entry," Journal of Spacecraft and Rockets, Vol. 45, No. 2, 2008.

${ }^{8}$ Johnson, H., Candler, G., "Hypersonic Boundary Layer Stability Analysis Using PSE-Chem," 35th AIAA Fluid Dynamics Conference and Exhibit, AIAA Paper 2005-5023, June 2005.

${ }^{9}$ Herbert, T. "Parabolized Stability Equations," Annual Review of Fluid Mechanics, Vol. 29, p245-283, 1997.

${ }^{10}$ Wright, M., Candler, G., "Data-Parallel Line Relaxation Method for the Navier-Stokes Equations," AIAA Journal, Vol. 36, Number 9, September 1998.

${ }^{11}$ Bertin, J., Periaux, J., Ballmann, J., “Advances in Hypersonics: Defining the Hypersonic Environment,” Birkhuser, 1992.

${ }^{12}$ MacLean, M., Mundy,E., Wadhams, T., Holden, M., Johnson, H., and Candler, G., "Comparisons of Transition Prediction Using PSEChem to Measurements for a Shock Tunnel Environment," 37th AIAA Fluid Dynamics Conference \& Exhibit, AIAA Paper 2007-4490, June 2007.

${ }^{13}$ MacLean, M., Wadahms, T., Holden, M., and Johnson, H., "Ground Test Studies of the HIFiRE-1 Transition Experiment Part 2: Computational Analysis," Journal of Spacecraft and Rockets, Vol. 45, No. 6, 2008.

${ }^{14}$ Anderson, J., "Hypersonics and High Temperature Gas Dynamics," McGraw-Hill, 1989. 